\title{
Beyond vibrationally mediated electron transfer: interfacial charge injection on a sub-10-fs time scale
}

\author{
Robert Huber ${ }^{\mathrm{a}}$, Jacques E. Moser ${ }^{\mathrm{b}}$, Michael Grätzel ${ }^{\mathrm{b}}$ and Josef Wachtveitl ${ }^{\mathrm{a}}$ \\ ${ }^{a}$ Institut für Physikalische und Theoretische Chemie, Marie-Curie-Str. 11, Goethe Universität \\ Frankfurt, 60439 Frankfurt am Main, Germany. \\ ${ }^{\mathrm{b}}$ Laboratory for Photonics and Interfaces, Institute of Molecular and Biological Chemistry, Ecole \\ Polytechnique Fédérale de Lausanne, CH-1015 Lausanne, Switzerland.
}

\begin{abstract}
The electron transfer (ET) from organic dye molecules to semiconductor-colloidal systems is characterized by a special energetic situation with a charge transfer reaction from a system of discrete donor levels to a continuum of acceptor states. If these systems show a strong electronic coupling they are amongst the fastest known ET systems with transfer times of less than $10 \mathrm{fs}$. In the first part a detailed discussion of the direct observation of an ET reaction with a time constant of about $6 \mathrm{fs}$ will be given, with an accompanying argumentation concerning possible artifacts or other interfering signal contributions. In a second part we will try to give a simple picture for the scenario of such superfast ET reactions and one main focus will be the discussion of electronic dephasing and its consequences for the ET reaction. We show that the actual ET process can be understood as a kind of dispersion process of the initially located electron into the colloid representing a real motion of charge density from the alizarin to the colloid.
\end{abstract}

Keywords: electron transfer, ultrafast, femtosecond, dye, semiconductor, titanium dioxide

\section{INTRODUCTION}

Sensitization of wide band gap semiconductors by organic dye molecules is a well established technique with widespread applications like the photographic process, detoxification and purification of water [1], and recently also solar energy to electricity conversion [2-9]. Considerable physical interest in these dye/semiconductor systems surely arises from the special energetic situation, allowing ultrafast electron transfer from a system with discrete energy levels on the donor side, into a continuum of energy levels on the acceptor side. In our case the elementary reaction can be written as

$$
S+h v \rightarrow S^{*} \rightarrow S^{+}+e_{\text {surf }}^{-}\left(\mathrm{TiO}_{2}\right) \rightarrow S^{+}+e_{C B}^{-}\left(\mathrm{TiO}_{2}\right)
$$

where the dye, attached to the colloidal surface is excited from the ground state $\mathrm{S}$ to the excited state $\mathrm{S}^{*}$, energetically situated above the conduction band edge of the semiconductor. The excited dye molecule ( $\mathrm{S}^{*}$ ) then acts as electron donor and electron transfer to surface states of the semiconductor and subsequently to the conduction band ( $\left.\mathrm{e}_{\mathrm{cb}}\right)$ occurs [10-31]. The dye molecule remains as cation $\mathrm{S}^{+}$. The back reaction and charge recombination to the ground state of the dye depends on the solvent, the presence/absence of electrolytes, the morphology of the $\mathrm{TiO}_{2}$ and also on the amount of adsorbed cations on the semiconductor surface [32]. The time scales for the back reaction vary from sub-picosecond up to milliseconds $[5,8,10,11,30,33-44]$. A detailed theoretical treatment of interfacial electron transfer is complicated, since reliable molecular models for the interface are needed and the presence of surface states or structural inhomogeneities have to be considered. Only recently, reasonable agreement between experiment and theory is reported for interfacial electron transfer reactions $[45,46]$. Nevertheless, due to the special energetic situation of a continuous band of acceptor levels, an activationless electron transfer can be assumed (Figure 1). According to Marcus theory, the only remaining parameter determining the injection rate should be the electronic coupling matrix element $\mathrm{V}^{2}$, representing the electronic overlap integral between donor and acceptor state. Whereas for weakly coupled systems injection times of $100 \mathrm{fs}-10 \mathrm{ps}[10,11,16,29,30,47,48]$ were found, it is not yet clear, if systems like alizarin $/ \mathrm{TiO}_{2}$ which show a very large shift in the absorption band upon coupling undergo a localized excitation ( $\mathrm{S} \rightarrow \mathrm{S}^{*}$ ) or if the excitation process itself transfers an electron from the HOMO of the dye molecule directly into the conduction band of the semiconductor. Here, we report the observation of an ultrafast electron transfer for this system on a sub-10- 
femtosecond timescale and propose that the sequence of reaction steps as indicated in equation (1) is still valid in the strong coupling case. A simple physical model will be used at the end of this paper to explain the scenario of such a superfast reaction.
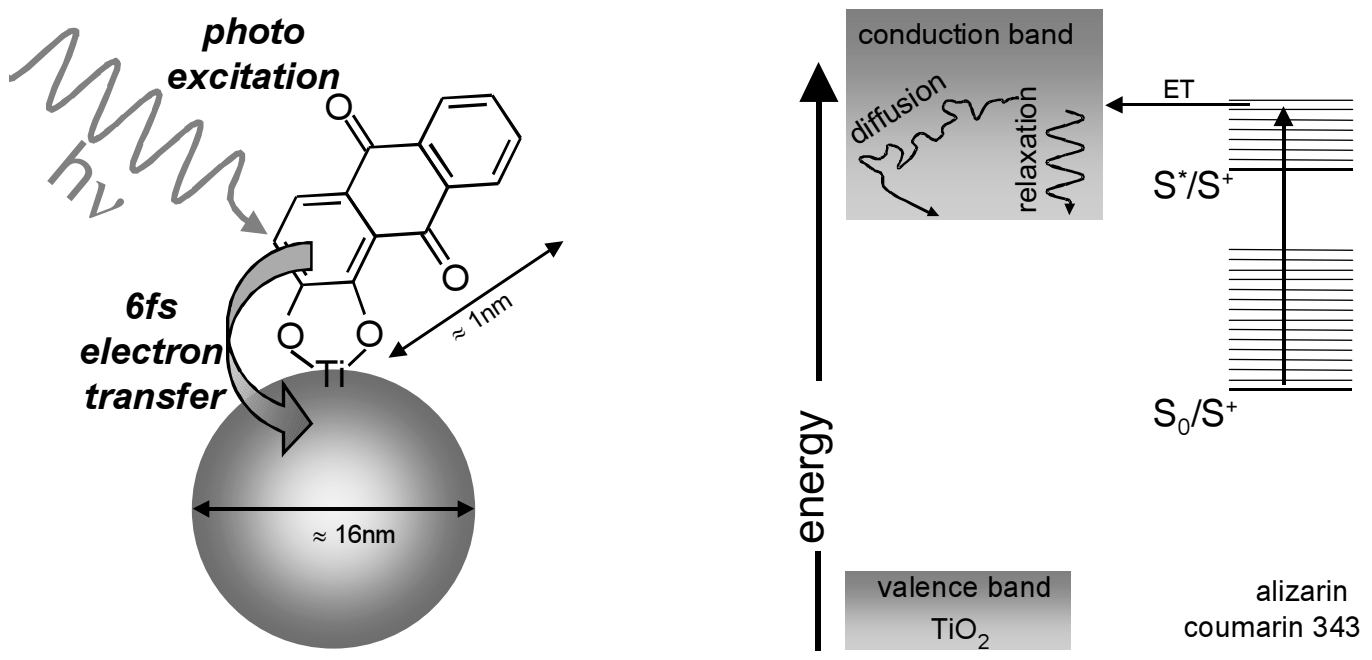

Fig. 1: (left:) Schematic drawing of the investigated system, an alizarin molecule is linked via two hydroxyl groups to a Ti-atom at the surface of the colloid; (right:) energetic situation, the excited state of alizarin lies above the edge of the $\mathrm{TiO}_{2}$ conduction band and therefore electron transfer after photo-excitation can occur.

\section{EXPERIMENTAL SECTION}

\section{Sample preparation}

Measurements were performed on samples containing alizarin coupled either onto the surface of $\mathrm{TiO}_{2}$ or $\mathrm{ZrO}_{2}$ colloids, respectively. The systems were diluted in methanol to final concentrations of $0.5 \mathrm{mM}$ alizarin on $10 \mathrm{~g} / \mathrm{l} \mathrm{TiO}{ }_{2}$ colloids. The colloidal nanoparticles of $\mathrm{TiO}_{2}$ were prepared as previously described via hydrolysis of $\mathrm{TiCl}_{4}$ in cold water and further dialysis of the sol. No stabilizing polymer was used. The average diameter of oxide particles was about $16 \mathrm{~nm}$, as measured by dynamic light scattering. $\mathrm{ZrO}_{2}$ colloidal dispersions were prepared by hydrolysis of zirconium chloride in methanol. $10 \mathrm{~g} \mathrm{ZrCl}{ }_{4}$ powder (Alfa) were dissolved in $150 \mathrm{ml}$ methanol. The solution was cooled to $0{ }^{\circ} \mathrm{C}$, acidified by $0.5 \mathrm{ml}$ conc. $\mathrm{HCl}, 4 \mathrm{ml}$ of $\mathrm{H}_{2} \mathrm{O}$ were added. After $2 \mathrm{~h}$ of stirring at room temperature, the colloid was boiled under reflux for $2-3 \mathrm{~h}$. The concentrated methanol-sol $(150 \mathrm{ml})$ was finally diluted in pure water $(700 \mathrm{ml})$ to yield a transparent colloidal solution containing typically $6 \mathrm{~g} / \mathrm{ZrO}_{2}$ particles and ca. $18 \mathrm{vol}$. \% methanol. The hydrodynamic diameter of the $\mathrm{ZrO}_{2}$ colloidal particles determined by quasi-elastic light scattering was $10-20 \mathrm{~nm}$.

\section{Femtosecond spectrometer}

The spectrometer for the transient absorption measurements is sketched in Fig. 2. The pump pulses (attenuated to $0,2 \mu \mathrm{J}$ at $495 \mathrm{~nm}$ ) were provided by a noncollinear optical parametric amplifier (NOPA) [49], pumped with $50 \mu \mathrm{J}$ pulses at $400 \mathrm{~nm}$ of the second harmonic of a homebuilt regenerative amplifier system $(1 \mathrm{kHz}, 1 \mathrm{~mJ}, 800 \mathrm{~nm})$. The NOPA was seeded with a white light supercontinuum, generated in a calcium fluoride plate [50]. The resulting pulse to pulse energy fluctuations of the NOPA were less than $1 \% \mathrm{rms}$ at pulse to pulse energy fluctuations of $<0.5 \%$ of the regenerative amplifier system at $800 \mathrm{~nm}$. The pulse duration of the NOPA output after compression was measured by a homebuilt intensity autocorrelator (second harmonic generation in a $25 \mu \mathrm{m}$ thick BBO) with material generating the same dispersion in the beam path from the NOPA to the autocorrelator than from the NOPA to the sample. The pulse compression of the NOPA pulses was performed by a standard prism compressor $\left(\mathrm{SiO}_{2}, 60^{\circ}\right)$. The pulse duration of the compressed NOPA output was measured to be about $19 \mathrm{fs}$ at a wavelength of $495 \mathrm{~nm}$ at the location of the sample. The diameter of the excitation spot inside the cuvette was $80 \mu \mathrm{m}$, the optical pathlength through the sample was $50 \mu \mathrm{m}$. 
The probe pulses were provided by supercontinuum (SC) generation with about $3 \mu \mathrm{J}$ of the $800 \mathrm{~nm}$ fundamental of the regenerative amplifier in a $\mathrm{CaF}_{2}$ plate. The low chirp of the $\mathrm{SC}$ generated in $\mathrm{CaF}_{2}[50]\left(<25 \mathrm{fs}^{2}\right.$ at $500 \mathrm{~nm},<17 \mathrm{fs}^{2}$ at $700 \mathrm{~nm}$ ) combined with a dispersion reduced setup was the prerequisite for a high temporal resolution. The SC is imaged via all reflective optics into the sample with a focal diameter of $40-50 \mu \mathrm{m}$, depending on the wavelength. The cross correlation functions, determined by fitting the coherent signals at the delay time zeros [51] were found to be in well agreement with measurements of two photon absorption (see [52]) and numerical summation of the pulse lengthening contributions which are: a) group velocity mismatch between pump pulse and probe pulse b) chirp of the SC c) non-collinearity angle between pump and probe (about $1^{\circ}$ internal). As shown in Figure 2 , the apparatus functions were 20-30 fs (fwhm) for $400 \mathrm{~nm}<\lambda<620 \mathrm{~nm}$, and $<50 \mathrm{fs}$ for the rest of the investigated wavelengths in the experiments reported here. The detection and data acquisition was performed as described in [53, 54]. Averaging about 4000 shots per scan and 16 scans per measurement, we were able to achieve a signal to noise ratio up to $10^{5}$ in transient transmission changes for the main part of the spectrum depending on the opacity of the sample.
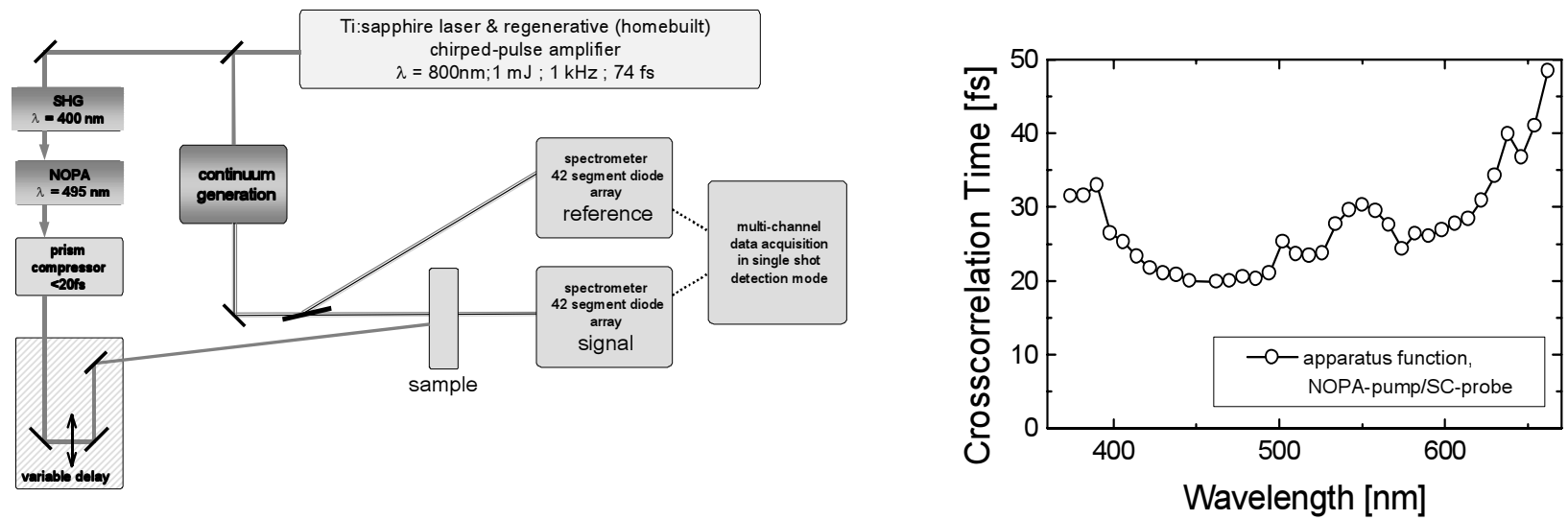

Fig. 2: (left:) Experimental set-up of the NOPA-pump/white light-probe spectrometer; (right:) wavelength dependent apparatus function of the set-up.

A precise determination of the delay time zeros, the prerequisite for a reliable deconvolution of the measured transients, was realized in two different, independent ways: a) the two photon absorption signal, arising when pump and probe photons pass a long pass filter glass simultaneously b) fitting the coherent signals at the delay time zero, recorded in pure methanol as described in [51]. In both cases, the determined delay time zeros, varying with the probing wavelength, were fitted using the Sellmeier equation with the known amount of dispersive material in the beam path of the probing SC. This fitting procedure further reduces of the uncertainty in the specific delay time zeroes. The error can be estimated from the discrepancy between the measured values and the fit curve, and was found to be less than 2 fs. To detect a possible drift of the delay time zeros with time, measurements of the pure solvent have been carried out before and after the actual measurement of the sample. This also allows to identify identical temporal signatures of the transients caused by coherent effects at the delay time zero in both the solvent and the sample. Thus we were able to verify that the influence of the sample on the dispersion and consequently on the group velocity is negligible. A measurement of a colloidal solution of $\mathrm{TiO}_{2}$ without alizarin has been carried out to check the potential signal originating from the sole colloids, with regard to direct multiphoton interband excitation by the pump pulse. Besides the coherent signal at the delay time zero, there was no further kinetic contribution found. The pure solvent data were used to subtract the coherent electronic signal at the delay time zero.

The analysis of temporal evolution of the transient absorption spectra, especially the spectral assignment of the occurring transient species was performed by a software package, developed at our institute, supplying a fast Marquardt downhill algorithm for a global fit of the recorded data, optimizing simultaneously $\mathrm{n}$ global decay times for all recorded transients at once. 


\section{SUB-10-FS CHARGE INJECTION}

\section{Spectroscopic properties of the samples}

To support the following detailed discussion of the transient dynamics first a characterization of the stationary properties of the samples is given in Fig. 3. The plotted spectra show the absorption for free alizarin in solution, for alizarin coupled onto $\mathrm{TiO}_{2}$ as well as on $\mathrm{ZrO}_{2}$ and for the alizarin cation. The spectrum of the alizarin cation was derived from nanosecond experiments by spectral subtraction. The absorption properties of an electron, injected in the conduction band of the $\mathrm{TiO}_{2}$ is also given and appears as flat unstructured spectrum from approximately $400 \mathrm{~nm}$ up to wavelengths of more than $800 \mathrm{~nm}$. The spectrum of the electron is stretched approximately by a factor of 5 for better visibility. It can be well seen, that the absorption of the alizarin cation exceeds the absorption of the alizarin ground state for wavelength $>600 \mathrm{~nm}$, but is considerably smaller in the wavelength region around $470 \mathrm{~nm}$. For wavelengths $>$ $650 \mathrm{~nm}$ the absorption of the electron becomes the dominant contribution. Therefore it is possible to distinguish all involved species by their spectral properties in the region from $350 \mathrm{~nm}$ to $700 \mathrm{~nm}$. Fig. 3 (right) shows the excited state spectra of alizarin on $\mathrm{ZrO}_{2}$ as well as alizarin on $\mathrm{TiO}_{2}$. In the case of $\mathrm{ZrO}_{2}$ as semiconductor colloid, where, due to the larger band gap no electron injection into the conduction band can occur, the calculation of the excited state spectrum by spectral subtraction is straight forward. For the calculation from the spectral signature of the difference absorption at $1 \mathrm{ps}$ the contributions of the ground state bleach and stimulated emission were subtracted. Even if this procedure does not reproduce all details of the spectrum, it is possible to characterize the main absorption properties of the excited state of coupled alizarin, especially the broad absorption band occurring at wavelengths $<450 \mathrm{~nm}$. The procedure for the deconvolution of the excited state spectrum of alizarin on $\mathrm{TiO}_{2}$ will be discussed later.
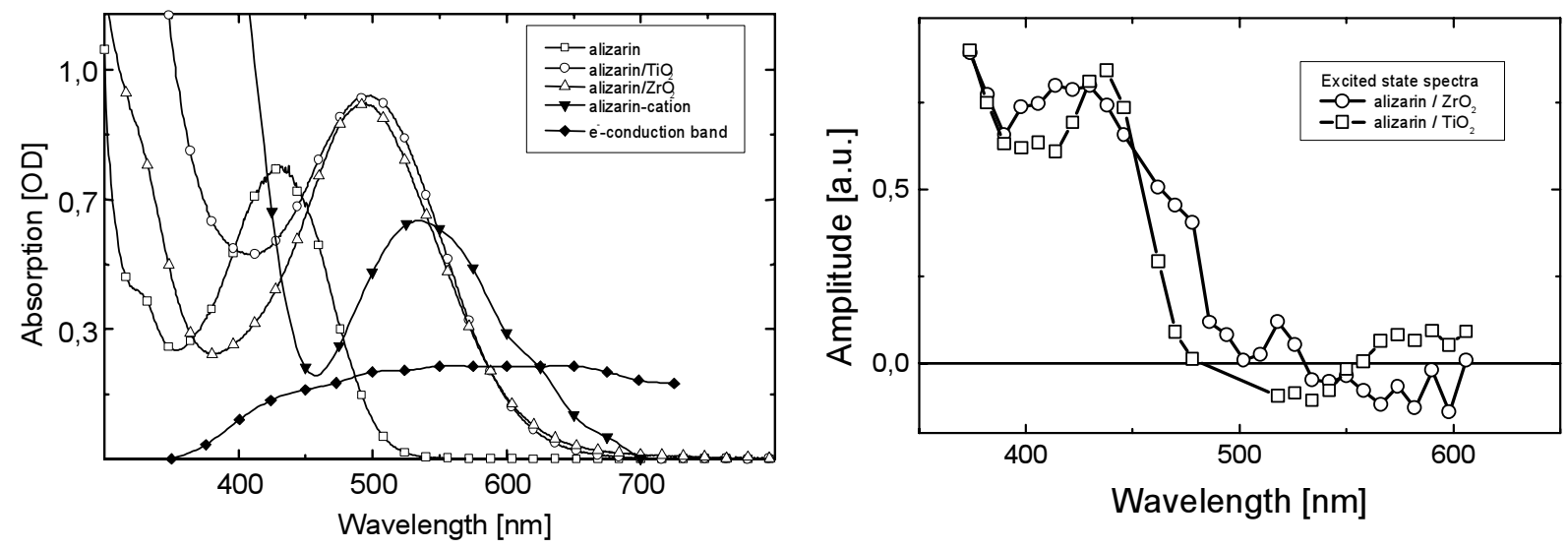

Fig. 3: (left:) Stationary spectra of alizarin; (right:) excited state spectra of alizarin on $\mathrm{TiO}_{2}$ and $A$ lizarin on $\mathrm{ZrO}_{2}$.

Transients at isosbestic points

For the investigation of the superfast reaction an analysis at the spectral positions without contributions of slower reactions will be performed. Such spectral positions are the two isosbestic points for the recombination reaction between the injected electron and the dye cation, marked in Fig. 4 (left, circles). As we showed [10], for time scales of $>100 \mathrm{fs}$ the reaction dynamics is dominated by this back reaction and therefore no, or only a minor spectral evolution can be recognized. Only a spectrally independent recovery of the ground state absorption is observed up to nanoseconds and longer. So one way to address the superfast ET reaction is the observation of the transient absorbance change at the spectral position of one of the isosbestic points where no slow reaction interferes with the fast kinetic. 

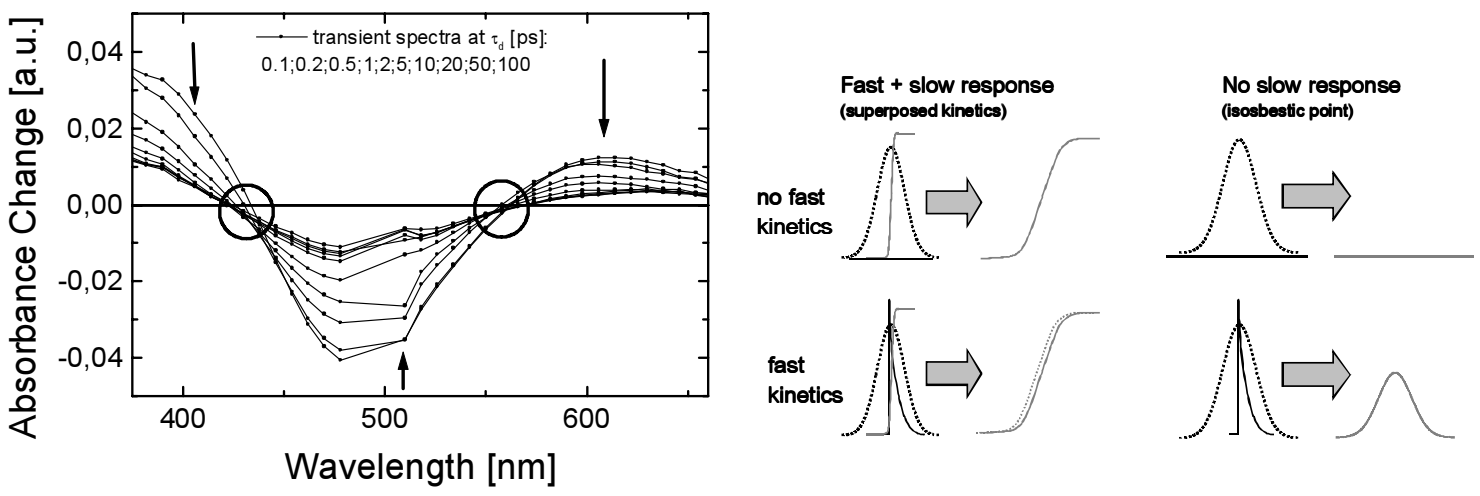

Fig. 4 (left): Transient spectra for alizarin/ $/ \mathrm{TiO}_{2}$ for delay times from 100 fs to $100 \mathrm{ps}$, the circles mark the isosbestic points; (right): sketch of superposed signals of a fast and a slow kinetic component - schematic drawing illustrating the advantage of a fit procedure at an isosbestic point.

Fig. 5 (left) shows transient data at a probe wavelength of $422 \mathrm{~nm}$, one of the isosbestic points. As this is no perfect isosbestic point, the data were numerically fitted by a biexponential decay with decay times of $6 \mathrm{fs}$ and one infinite modeling the superfast decay and the plateau. The numerical stability of the fitting procedure should be visualized by two additional curves, the faster one representing a decay with a constant of $3 \mathrm{fs}$ and a slower one, representing a $9 \mathrm{fs}$ decay. For both additional curves a quantitative deviation from the experimental data can be seen. A detailed discussion of the single trace fit can be found in ref. [10].

\section{Global fit of transient data}

To verify the superfast kinetic component and to exclude possible artifacts contributing to the signal, a global fit of all transients was performed simultaneously. Thereby a defined number of decay times is optimized keeping the same value for all wavelength channels. The global fitting procedure in our case shows less numerical stability as there are numerous channels where the superfast component occurs only with a small amplitude. Therefore the resulting global time constants vary between 5 and $7 \mathrm{fs}$, depending on the start values.

For an estimation of the quality of the global fits, Fig. 6 shows the results for various wavelengths channels throughout the entire investigated spectrum. The fit shows one fast decay with a time constant of $6.5 \mathrm{fs}$ and two slower ones, representing the succeeding long time evolution. Obviously the transients can be described quite well by this model function and no prominent systematic deviation to the data can be observed. For a more detailed analysis of the spectral distribution of the signal amplitude of the superfast component Fig. 5 (right) shows a decay related spectrum, the wavelength dependent linear prefactors of the fit curves for the $6.5 \mathrm{fs}$ time constant. A positive sign in this amplitude spectrum means a decay, a negative sign an increase. The value corresponds with the amplitude of the decay for the particular wavelength.
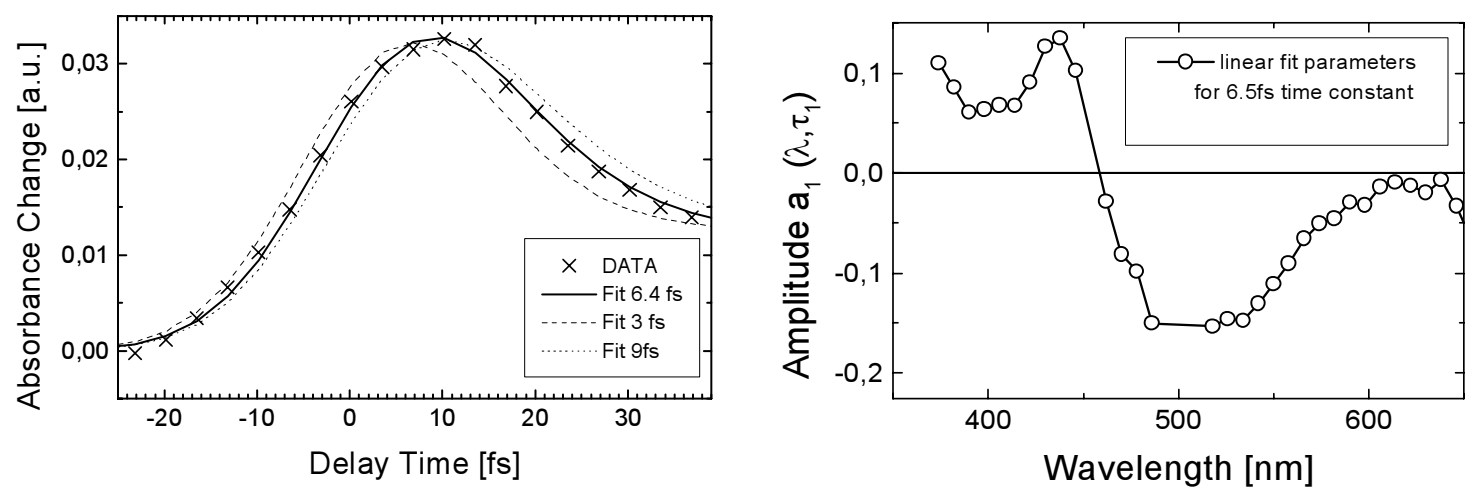

Fig. 5 (left): Numeric fit for transient at $422 \mathrm{~nm}$ and obvious deviation of fits with faster ( $3 \mathrm{fs}$ ) and slower (9 fs) kinetic components; (right): decay related spectrum of 6.5 fs time constant. 

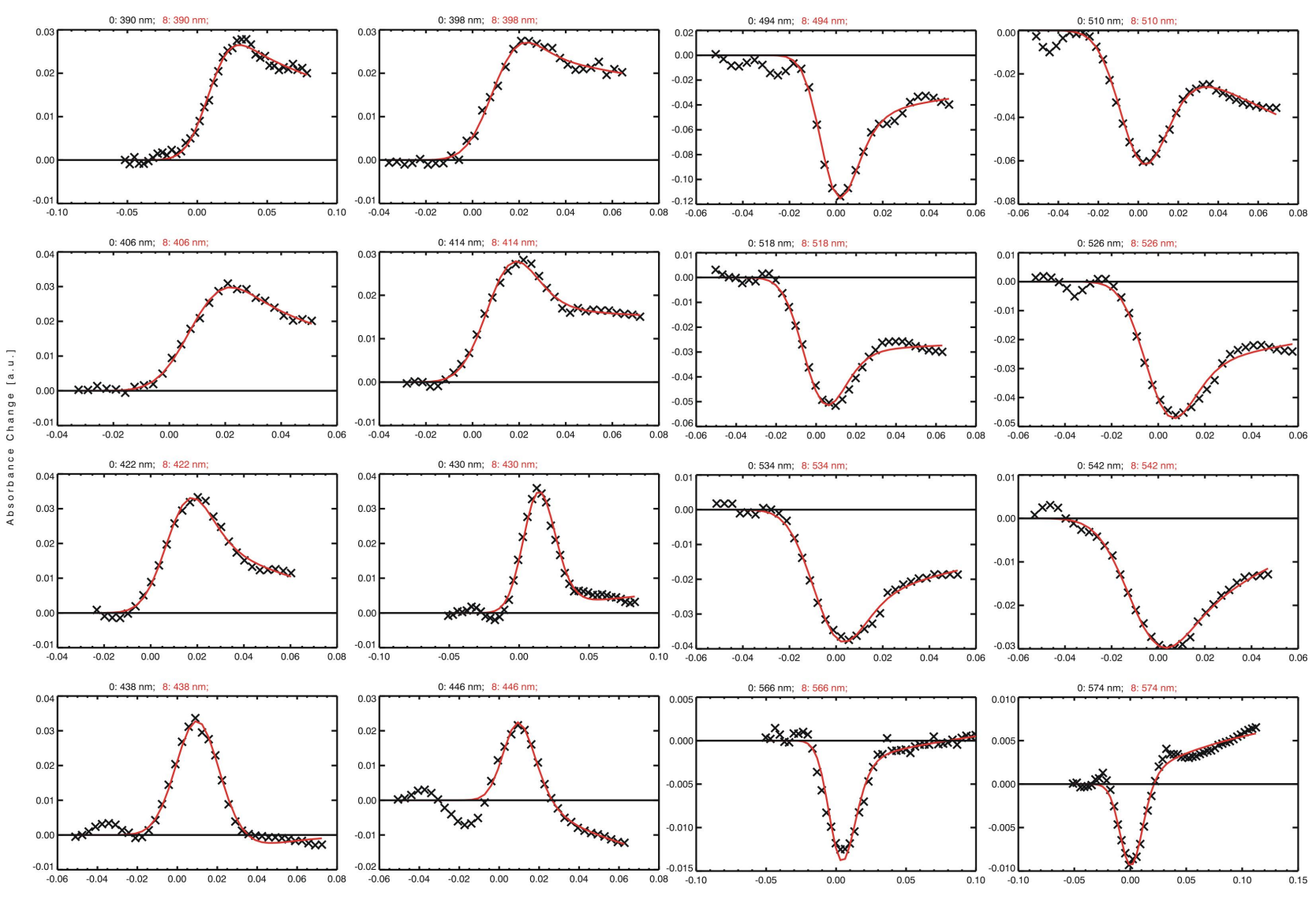

Delay Position [ps]

Delay Position [ps]

Fig. 6: Single transients for different wavelengths and the fit curves resulting from the global fitting procedure: obviously the ultrafast kinetics can be observed over a wide spectral range with a significant amplitude; the perturbation of the signal by the "coherent artifact" at the delay time zero, where pump and probe pulse overlap, can be identified to have a negligible contribution, a minor oscillation can only be seen at $438 \mathrm{~nm}, 446 \mathrm{~nm}, 494 \mathrm{~nm}$ and $510 \mathrm{~nm}$ ).

\section{Possible Artifacts}

Even at first sight one can classify the shape of the decay related spectrum as "physical" with no discontinuity or very sharp structures which would not be expected for a superfast reaction of less than $10 \mathrm{fs}$. Furthermore possible artifacts can be ruled out. Two quantum absorption between a pump and a probe photon as reason for the peak like signal would only lead positive signals, to a transient absorption increase, transient decreases in absorption, as seen for wavelengths $>500 \mathrm{~nm}$ could not be due to two quantum absorption. A stimulated Raman processes could also be envisioned as possible reason for the observed ultrafast dynamics. If the frequency difference between the pump and the probe pulse corresponds to a vibrational transition of the solvent, and if the pulses are shorter than the vibrational dephasing time $T_{2}$ of the solvent, two peaks with different signs and with the characteristic bandwidth of the pump pulse should be visible in the transient absorption experiments: Raman gain at the low frequency side and Raman loss at the high frequency side of the pump pulse. The linear amplitudes of the global fitting procedure for the time constant $\tau_{1}$ (Figure 5, right) indeed exhibit a significant positive peak at $438 \mathrm{~nm}$ with an energy difference of $2629 \mathrm{~cm}^{-1}$ to the pump pulse $(495 \mathrm{~nm})$. However, since no prominent band is found in the spectrum of methanol and the corresponding negative peak at $569 \mathrm{~nm}$ is missing, stimulated Raman processes can be ruled out as origin for the ultrafast dynamics. Signal contribution from the solvent as a cause for the superfast signal can also be ruled out, as in the transient absorption measurements neither for methanol nor for a colloidal $\mathrm{TiO}_{2}$ solution without coupled alizarin ultrafast transient absorption changes in this spectral region were observed. Furthermore, the coherent signal of the solvent 
around delay time zero was typically small and could be well subtracted from the sample data. A further possible effect causing a signal on the sub $10 \mathrm{fs}$ timescale could be the dephasing of the excited state after the photo-excitation with no subsequent electron injection. This effect can be ruled out, as the decay associated spectrum in Fig. 5 (right) shows contributions of the superfast component up to $650 \mathrm{~nm}$. At this spectral position neither the ground state (Fig. 3, left) nor the excited state (Fig. 3, right) show absorption in contrast to the electron inside the $\mathrm{TiO}_{2}$ and the cation. If the signal would only be caused by the excited state dephasing no superfast transient absorption signal at $>600 \mathrm{~nm}$ would be observed. The next effect that should be considered is the coherent interaction between pump and probe pulse e.g. cross phase modulation. These signals usually cause oscillatory contributions in the transients for the time range where pump and probe pulse overlap. It should be pointed out, that the signals of all transients in Fig. 6 show one dominant peak and only few minor oscillatory contributions. Furthermore for low chirped pulses typically should have an antisymmetric signature in time and in wavelength (frequency) a feature also absent in the observed transients in our system. Possible other physical processes, like ultrafast molecular reorganization with a dvnamic shift in the spectral signatures can also be excluded, because they would result in a characteristic anti-symmetric feature in the decay related spectra, which was not observed.

\section{Analysis of the decay related spectra}

As the previous discussion revealed, the observed superfast signal should be addressed to a real physical reaction. In the following a detailed analysis will demonstrate that the signature of the amplitude is in good agreement with the proposed model of photo-excitation and a subsequent electron injection. As described above, the optimized global time constant is $\tau_{1}=6.5 \mathrm{fs}$. The spectral signature of the linear parameter in Figure 6 for the decay time $\tau_{1}$ can now be analyzed to assign the different species to components of the decay associated spectrum. For comparison, the absorption spectra of the alizarin cation $\mathrm{S}^{+}$, the alizarin ground state $\mathrm{S}^{0}$ and the absorption of the electron in the colloid's conduction band (Fig. 3) should be compared to the decay associated spectrum in Fig. 5 (right). For wavelengths $<460 \mathrm{~nm}$ the decay associated spectrum can be assigned to the cation formation and to the rise of absorption due to the injected electron. The pronounced minimum between $500 \mathrm{~nm}$ and $550 \mathrm{~nm}$ reflects the cation absorption, whereas the flat unstructured region at $\lambda>580 \mathrm{~nm}$ is typical for the injected electron. The positive amplitudes for wavelengths $\lambda<460 \mathrm{~nm}$ arises from the excited state absorption, which is partially compensated for $\lambda<430 \mathrm{~nm}$, again in good agreement with the increased absorption of the $\mathrm{S}^{+}$spectrum for $\lambda<450 \mathrm{~nm}$ in Fig. 3. In conclusion, the spectral characteristics of the fast time constant $\tau_{1}$ fits perfectly with an electron injection process in the complete range of wavelengths between $350 \mathrm{~nm}$ and $650 \mathrm{~nm}$ reflecting cation formation, the vanishing $\mathrm{S}^{*}$ state and the electron in the semiconductor.

\section{$\mathrm{S}$ * spectrum of alizarin/ $\mathrm{TiO}_{2}$}

For a further confirmation of the assignment of the instantaneous absorbance increase to excited state absorption, we compare the excited state spectrum of alizarin on $\mathrm{TiO}_{2}$ to the one of alizarin on $\mathrm{ZrO}_{2}$. We showed in ref. [11], that for the system alizarin on $\mathrm{ZrO}_{2}$, the excited state of alizarin is repopulated after $1 \mathrm{ps}$ to about $80 \%$. This means, that the transient spectrum of alizarin adsorbed on $\mathrm{ZrO}_{2}$ for $\mathrm{t}_{\mathrm{D}}=1 \mathrm{ps}$ should look similar to the initial transient spectrum of alizarin on $\mathrm{TiO}_{2}$ at delay time 0 . Because of the fast decay of the excited state in the latter case, the transient spectrum at $\mathrm{t}=0$ has to be deconvoluted from the decay related spectra. This can be done by simply summing up the linear fitting amplitudes for the different decay times for each channel of the global fit, resulting in the transient spectrum at the delay time zero that would be measured by a setup with infinite temporal resolution. From the transient spectra, the excited state spectra are then calculated by spectral subtraction of the ground state absorption. Fig. 3 (right) shows both $\mathrm{S}^{*}$ spectra, for the systems alizarin $/ \mathrm{TiO}_{2}$ and alizarin $/ \mathrm{ZrO}_{2}$. The qualitative good agreement provides a very strong evidence for an assignment of the $6 \mathrm{fs}$ time constant to the decay of the excited state $\mathrm{S}^{*}$. The broad spectra shape of the $\mathrm{S}^{*}$ agrees also well with the bandwidth expected from energy/time uncertainty. A $6 \mathrm{fs}$ lifetime leads to a spectral broadening of less than $20 \mathrm{~nm}$ for a central wavelength of $450 \mathrm{~nm}$. Obviously there is no conflict between the lifetime of the $\mathrm{S}^{*}$ state and the absorption bandwidth.

From the previous discussion it can be concluded, that the observed transients really reflect a transition from the excited state of the alizarin to a charge separated state. Directly after excitation the complete spectral signature reflects the coupled alizarin molecule in the $\mathrm{S}^{*}$ state and on a time scale of $6 \mathrm{fs}$ the signature changes to assume the spectral shape of the charge separated state - the alizarin cation and an injected electron. In the following a simple picture describes the scenario of such a superfast ET. 


\section{PHYSICAL MODEL OF SUPERFAST ET REACTION - VIBRONIC MEDIATION AND ELECTRONIC DEPHASING}

In the previous chapter we assigned the observed transient absorbance changes to a superfast injection of an electron from the alizarin molecule to the $\mathrm{TiO}_{2}$ colloid. In the following the occurrence of such a fast charge transfer reaction is discussed. Fig. 7 shows the main difference between a standard molecular (left) electron transfer regime and the situation at a dye/semiconductor interface (right). The crucial issue in the standard molecular ET, as treated by the Marcus theory, is the coupling between ET and molecular vibration. The ET itself can be understood as quantum mechanical tunneling process which is mediated by molecular vibrations. As illustrated in Fig. 7 for an ET after photoexcitation, the system in the excited state propagates in a classical sense on the potential energy surface along the reaction coordinate. This coordinate combines the influence of relevant molecular modes of the molecule as well as the solvent surrounding. The propagation expressing molecular vibrations can either be directly excited by an ultrashort laser pulse, generating a coherent superposition of molecular eigenmodes, or more commonly by thermal activation where the occupation of the different vibrational levels follows the Boltzmann distribution. In a microscopic view, treating the system like a classical oscillator within the potential surface of the excited state, the actual electron transfer process, i.e. the tunneling of the electron from the donor to the acceptor, can only occur, when the system crosses the intersection between the potential energy surfaces of the excited state and the charge separated state. This means, ET can occur only in the nuclear configuration of molecule and the surrounding solvent where the complete system has the same energy in the excited state as well as in the charge separated state. Each time the system crosses the intersection, ET happens with a certain probability. The transition probability is usually calculated by the Landau-Zener theory depending on the electronic coupling matrix element of the two electronic states involved. This energy matching mechanism can be sketched in a picture of quantum dots as periodic shift of the electronic energy level of the donor system to supply equal energy regarding the acceptor level (Fig. 7, left, inset). The electron transfer is mediated by molecular vibrations as key feature for an energy matching mechanism.
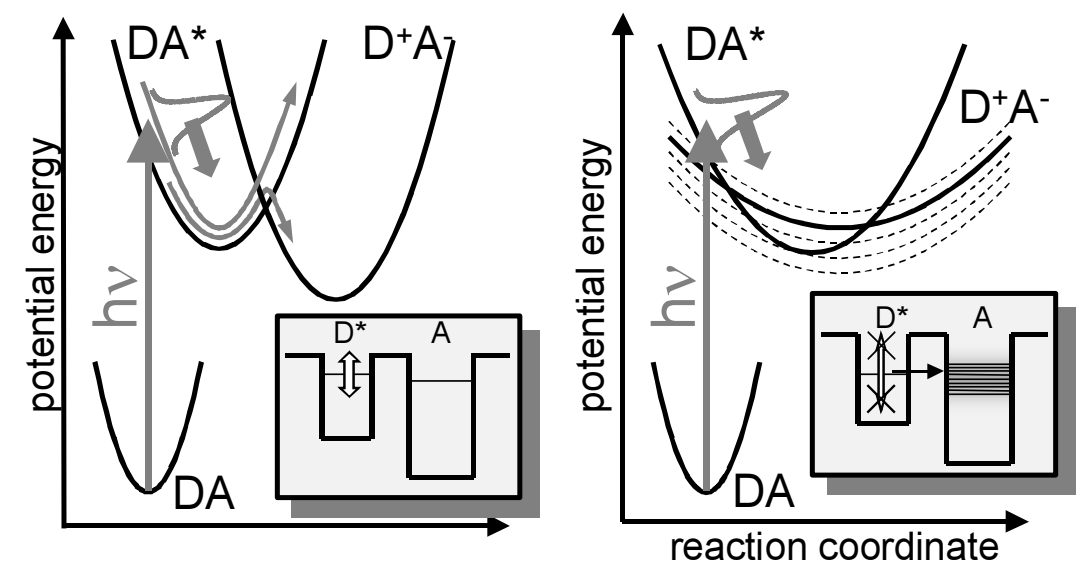

Fig. 7: The difference between classical molecular ET and molecule/semiconductor ET; (left:) in the classical ET as treated by the Marcus theory, the ET reaction is mediated by molecular vibrations as an energy matching mechanism; (right:) in the dye/semiconductor system no energy matching is required an therefore the observed injection times are much faster.

In the dye/semiconductor system a completely different situation arises. In the case of a semiconductor acting as acceptor with an energetically broad conduction band, no energy matching mechanism is needed. The energy of the donor level always matches energy states of the acceptor. The electron can undergo a transfer reaction in every configuration of the molecule and its solvent surrounding. There is no more coupling or mediation of the ET process by molecular vibrations necessary. As we deal here with a strongly coupled system, second order effects like the variation of the distance by molecular vibrations and therefore a modulation of the ET probability can be neglected. Furthermore a modulation of the density of states within the conduction band of the semiconductor will vary from molecule to molecule and so modulations of the transfer by molecular vibrations will hardly be detectable. 
A direct consequence of the decoupling of molecular vibration and ET reaction are the tremendously increased reaction rates of dye/semiconductor systems compared to pure molecular systems. For almost all of these systems ET time constants occur on an ultrafast time scale and lie in the range below 1 ps $[10,11,16,29,30,47,48]$. As for a quantum mechanical tunneling the only relevant remaining quantity for the injection time is the coupling between the donor and acceptor state. As this coupling is related to the overlap of the single wave function for the donor and the acceptor system, the distance between the donor and the acceptor system have a main influence on the coupling and the reaction rate. As shown in ref. [10] the excitation of the alizarin molecule already leads to a displacement of electron density toward the $\mathrm{TiO}_{2}$ surface, and therefore a strong electronic coupling and a very fast reaction can be expected. In the following the electron transfer in the alizarin/ $/ \mathrm{TiO}_{2}$ is modeled as system of two coupled quantum states for the donor as well as for the acceptor side.
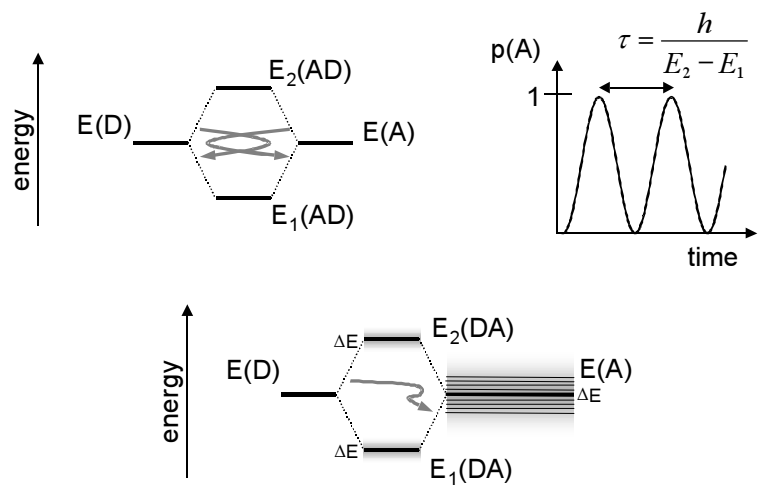

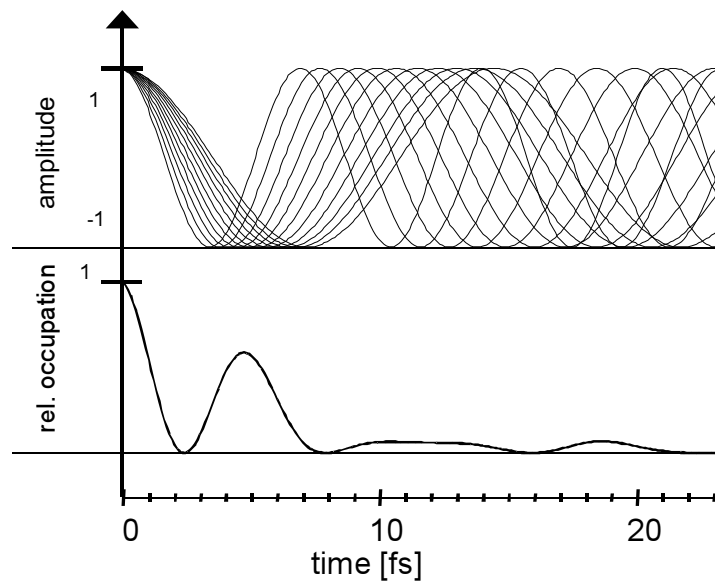

Fig. 8 (left): Quantum beats in isolated system of two coupled quantum states. Without dephasing, the population of the donor system D would lead to an infinite oscillation of population between donor and acceptor side (top). In the case of a broad continuum of acceptor states fast dephasing leads to effective net transfer of population (bottom). (right): Temporal evolution of a coupled quantum system with fast dephasing, amplitude (top), population (bottom).

To explain the net electron transfer rate, dephasing processes have to be taken into account. In Figure 8 (left) quantum beats in an isolated system of two coupled quantum states are shown. Neglecting dephasing the population of the donor system D would lead to an infinite oscillation of population between donor and acceptor side. Possible further reaction channels and the coupling to other quantum states would lead to a dephasing an to a stationary occupation finally resulting in a net transfer from $\mathrm{D}$ to $\mathrm{A}$. In the case of a broad continuum of acceptor states this dephasing is tremendously accelerated by the fact that many acceptor states with different energies are coupling to the donor state. This effect is sketched in Fig. 8 (right). As model for the alizarin/ $/ \mathrm{TiO}_{2}$ system a coupling of about $3000 \mathrm{~cm}^{-1}$ as calculated by the Mulliken-Hush equation for the coupled alizarin was assumed, an energy uncertainty of $\pm 1000 \mathrm{~cm}^{-1}$ reflects the spectral broadening of the absorption band upon coupling (Fig. 3, left) implying that this broadening is purely due to electronic dephasing. As a sketch in Fig. 8 (right) the resulting situation is shown. In the upper part the amplitudes of the wave functions are drawn, in the lower part the square of the sum of all oscillations. The sum represents the actual occupation of the donor state D. It can be clearly seen, that after about one period the system is completely dephased, the donor state is completely depopulated. The time scale of this process can be estimated to be somewhere below $10 \mathrm{fs}$ assuming an averaged curve on the oscillation decay in Fig. 8 (right). It should be noted, that the observed decoherence is no artifact reflecting the electronic dephasing of the excited state and ground state population after the photo-excitation. The dephasing described here concerns the quantum states of the excited system and the charge separated system reflecting the real charge transfer kinetics.

In conclusion in the proposed model for the interfacial electron transfer the expected decay time lies on the same time scale as the observed $6 \mathrm{fs}$ reaction. The proposed model of fast electronic decoherence is consistent with the broad band of electron acceptor levels and provides a good picture for the interpretation of the reaction. As the non-coherent superposition of wave functions not only describes the depopulation of the donor state but also the dispersion of electron density inside the $\mathrm{TiO}_{2}$ colloid, in coordinate space this picture would express the transfer of electron density 
localized near the alizarin to a wide spread distribution within the colloid. The observed kinetics can be unambiguously assigned to the electron transition from the alizarin to the $\mathrm{TiO}_{2}$.

\section{CONCLUSION}

In this paper, we describe the superfast ET from the organic dye molecule alizarin to the semiconductor colloid $\mathrm{TiO}_{2}$. The analysis of transient absorption measurements lead a physical model describing the actual situation for the $6 \mathrm{fs}$ charge transfer reaction. The key role of electronic dephasing between the excited state of alizarin and the charge separated states is proposed. The spectral characteristics of the process is used to distinguish this kind of dephasing from electronic dephasing of the excited state and the ground state after photo-excitation. The observations allow to conclude that the initial 6 fs reaction really reflects a spatial displacement of electron density from the vicinity of the alizarin to the $\mathrm{TiO}_{2}$.

\section{REFERENCES}

1. Wu, T., Liu, G. \& Zhao, J., J. Phys. Chem. 103, 4862-4867 (1999).

2. O'Regan, B. \& Schwartz, D.T., J. Appl. Phys. 80, 4749-4754 (1996).

3. $\quad$ Nazeeruddin, M.K., et al., J. Am. Chem. Soc. 115, 6382-6390 (1993).

4. Hagfeldt, A., Björksten, U. \& Grätzel, M., J. Phys. Chem. 100, 8045-8048 (1996).

5. O'Regan, B. \& Grätzel, M., Nature 353, 737-740 (1991).

6. $\quad$ Nazeeruddin, M.K., et al., Helv. Chim. Acta 73, 1788-1803 (1990).

7. Enea, O., Moser, J. \& Grätzel, M., J. Electroanal. Chem. 259, 59-65 (1989).

8. O'Regan, B., et al., J. Phys. Chem. 94, 8720-8726 (1990).

9. Bach, U., et al., Nature 395, 583-585 (1998).

10. Huber, R., et al. Ultrafast Phenomena XII. 2000. Berlin: Springer Verlag.

11. Huber, R., et al., J. Phys. Chem. 104, 8995-9003 (2000).

12. Huber, R., et al., J. Phys. Chem. B 106, 6494-6499 (2002).

13. Kamat, P.V., Prog. React. Kin. 19, 277-316 (1994).

14. Argazzi, R., et al., J. Phys. Chem. 101, 2591-2597 (1997).

15. Martini, I. \& Hartland, G.V., J. Phys. Chem. 100, 19764-19770 (1996).

16. Martini, I., Hodak, J.H. \& Hartland, G.V., J. Chem. Phys. 107, 8064-8072 (1997).

17. Martini, I., Hodak, J.H. \& Hartland, G.V., J. Phys. Chem. 102, 607-614 (1998).

18. Zimmermann, H. \& Boyn, R., Phys. Stat. Sol. 139, 533-545 (1987).

19. Moser, J. \& Grätzel, M., J. Am Chem. Soc. 106, 6557-6564 (1984).

20. Moser, J., et al., Helv. Chim. Acta 24, 1686-1690 (1985).

21. Hilgendorff, M. \& Sundström, V., Chem. Phys. Lett. 287, 5-6 (1998).

22. Hilgendorff, M. \& Sundström, V., J. Phys. Chem. 102, 10505-10514 (1998).

23. Ghosh, H.N., et al., J. Phys. Chem. 102, 10208-10215 (1998).

24. Ghosh, H.N., Asbury, J.B. \& Lian, T., J. Phys. Chem. 102, 6482-6486 (1998).

25. Tachibana, Y., et al., J. Phys. Chem. 100, 20056-20062 (1996).

26. Ellingson, R.J., et al., J. Phys. Chem. 102, 6455-6458 (1998).

27. Hannappel, T., et al., J. Phys. Chem. 102, 3651-3652 (1998).

28. Hannappel, T., Burfeindt, B. \& Storck, W., J. Phys. Chem. 101, 6799-6802 (1997).

29. Burfeindt, B., et al., J. Phys. Chem. 100, 16463-16465 (1996).

30. Wachtveitl, J., et al., Int. J. Photoenerg. 1, 153-156 (1999).

31. Heimer, T.A. \& Heilweil, E.J., J. Phys. Chem. 101, 10990-10993 (1997).

32. Pelet, S., Moser, J.E. \& Grätzel, M., J. Phys. Chem. 104, 1791-1795 (2000).

33. Haque, S.A., et al., J. Phys. Chem. 104, 538-547 (2000).

34. Tachibana, Y., et al., J. Photochem. Photobiol. 141, 215-220 (2001).

35. Nogueira, A.F., De Paoli, M.A. \& Montanari, I., J. Phys. Chem. 105, 7517-7524 (2001).

36. Ramakrishna, G., et al., J. Phys. Chem. 105, 12786-12796 (2001).

37. Ramakrishna, S., Willig, F. \& May, V., J. Chem. Phys. 115, 2743-2756 (2001). 
38. Moser, J. \& Grätzel, M., Chem. Phys. 176, 493-500 (1993).

39. Moser, J., Sol. Energ. Mater. Sol. Cells 38, 343-345 (1995).

40. Huber, R., et al., Chem. Phys. 285, 39-45 (2002).

41. Furube, A., et al., J, Phys. Chem. 107, 4162-4166 (2003).

42. Pelet, S., Gratzel, M. \& Moser, J.E., J. Phys. Chem. 107, 3215-3224 (2003).

43. Pan, J., et al., J. Am. Chem. Soc. 124, 13949-13957 (2002).

44. Schnadt, J., et al., Nature 418, 620-623 (2002).

45. Gao, Y.Q. \& Marcus, R.A., J. Chem. Phys. 113, $6351-6360$ (2000).

46. Gao, Y.Q., Georgievskii, Y. \& Marcus, R.A., J. Chem. Phys. 112, 3358-3369 (2000).

47. Asbury, J.B., et al., J. Phys. Chem. 105, 4545-4557 (2001).

48. Rehm, J.M., et al., J. Phys. Chem. 100, 9577-9578 (1996).

49. Wilhelm, T., Piel, J. \& Riedle, E., Opt. Lett. 22, 1494-1496 (1997).

50. Huber, R., et al., Opt. Commun. 194, 443-448 (2001).

51. Kovalenko, S.A., et al., Phys. Rev. A 59, 2369-2384 (1999).

52. Rasmusson, M., et al., Chem. Phys. Lett. 335, 201-208 (2001).

53. Nägele, T., et al., Chem. Phys. Lett. 272, 489-495 (1997).

54. Huber, H., et al., Chem. Phys. 197, 297-305 (1995). 